\title{
Urine miR-21-5p as a potential biomarker for predicting effectiveness of tadalafil in benign prostatic hyperplasia
}

\author{
Tomoaki Tanaka*,1, Akinori Minami ${ }^{1}$, Kouichiro Tashiro ${ }^{2,3}$, Naomasa Yoshida ${ }^{3}$, Akira \\ Tohda $^{4}$, Yasuo Yamakoshi ${ }^{5}$, Ryoji Yasumoto ${ }^{6}$, Shozo Sugita ${ }^{7} \&$ Tatsuya Nakatani ${ }^{1}$ \\ ${ }^{1}$ Department of Urology, Osaka City University Graduate School of Medicine, Osaka, Osaka, Japan \\ ${ }^{2}$ Department of Urology, Meijibashi Hospital, Matsubara, Osaka, Japan \\ ${ }^{3}$ Department of Urology, Yoshida Hospital, Hirakata, Osaka, Japan \\ ${ }^{4}$ Department of Urology, Moriguchi Ikuno Memorial Hospital, Moriguchi, Osaka, Japan \\ ${ }^{5}$ Department of Urology, Ishikiriseiki Hospital, Higashiosaka, Osaka, Japan \\ ${ }^{6}$ Yasumoto Nephrology-Urology Clinic, Osaka, Osaka, Japan \\ ${ }^{7}$ Department of Urology, Ohno Memorial Hospital, Osaka, Osaka, Japan \\ *Author for correspondence: tomoaki826@msic.med.osaka-cu.ac.jp
}

Aim: To investigate whether urine levels of miRNAs that regulate the function of endothelial cells are associated with effectiveness in benign prostatic hyperplasia (BPH) patients treated with a phosphodiesterase type 5 inhibitor, tadalafil. Patients \& methods: We measured urine levels of three miRNAs (miR-21-5p, miR-126-5p \& miR-155-5p) in 55 BPH patients before and after tadalafil administration to understand its effectiveness. Results: Baseline urine miR-21-5p level was an independent predictor of response to tadalafil in multivariate regression analysis (odds ratio: $0.28 ; 95 \% \mathrm{Cl}: 0.10-0.77 ; \mathrm{p}=0.014$ ). Receiver operator curve analysis revealed that baseline urine miR-21-5p could serve as a predictor of response (area under curve: 0.85; $95 \% \mathrm{Cl}: 0.75-0.95 ; \mathrm{p}<0.001)$. Conclusion: Urine miR-21-5p could serve as a biomarker in predicting response of tadalafil for $\mathrm{BPH}$.

Lay abstract: Tadalafil, a phosphodiesterase type 5 inhibitor, has a high potential for treating male lower urinary tract symptoms; however, there are few reports regarding which portion of the population is appropriate for treatment with tadalafil. This study showed that the baseline urine levels of miR-21-5p, an endothelium-associated miRNA, strongly correlates with response to tadalafil and have the potential to be a predictive biomarker of whether treatment with tadalafil is suitable.

\section{Graphical abstract:}

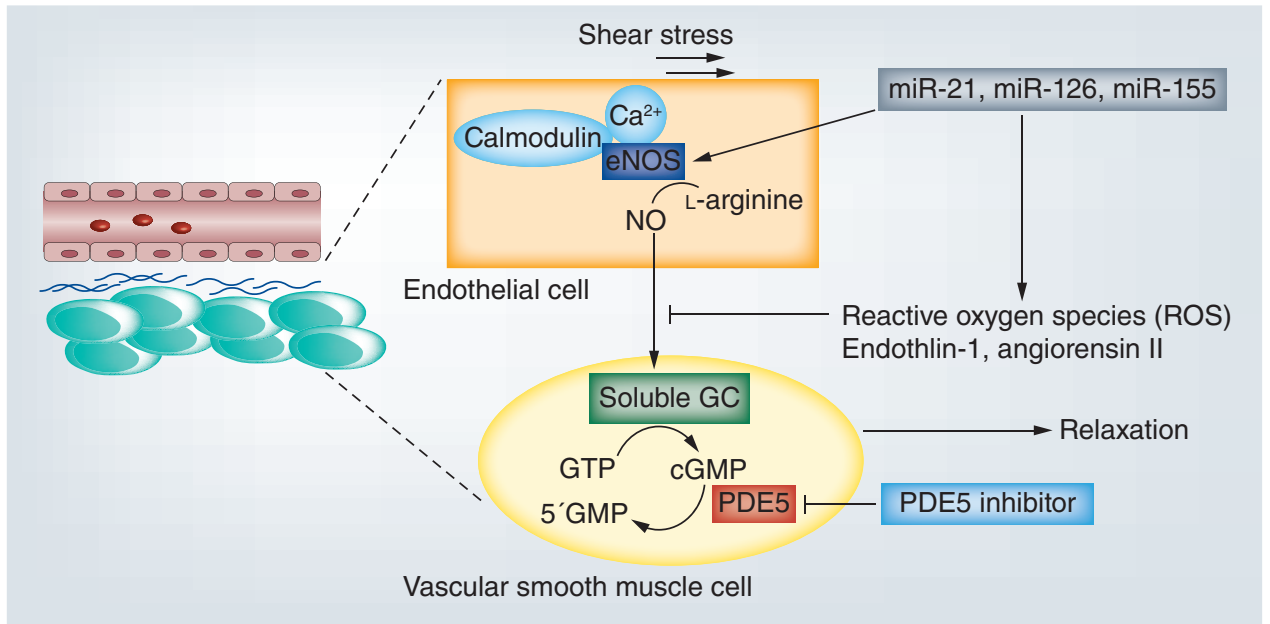

First draft submitted: 29 January 2018; Accepted for publication: 26 February 2018; Published online: 15 March 2018 
Keywords: biomarker $\bullet$ BPH $\bullet$ endothelial nitric oxide synthase $\bullet$ endothelium $\bullet$ LUTS • miR-21-5p • NO • PDE5 • tadalafil • urine

Male lower urinary tract symptoms (mLUTS) associated with benign prostatic hyperplasia (BPH) are a common complaint in senior adult men with a strong impact on quality of life (QoL), and substantial financial loss can result. The components of mLUTS include some symptoms, such as nocturia, increased urinary frequency, urgency, slow stream and sensation of incomplete emptying, in a voiding and bladder storage phase in urination. In general, some factors (e.g., insulin resistance, change in hormone balance, arterial sclerosis in pelvis, local inflammation) derived from aging and metabolic syndrome are believed to accelerate mLUTS with enlargement of prostate causing bladder outlet obstruction and bladder overactivity [1-3]. Although some medical treatments have been developed to target bothersome voiding or/and storage symptoms associated with mLUTS, one of the PDE5 inhibitors (PDE5-Is), tadalafil, is a brand-new drug for treatment of mLUTS/BPH and is recommended as a level A in worldwide clinical treatment guidelines owing to various excellent evidence [4,5]. PDE5-Is were reported to have some individual actions including the improvement of blood flow of pelvic viscera [6,7] and suppression of chronic prostatic inflammation [8], in addition to the release of bladder outlet obstruction [9] through the inactivation of PDE5/cyclic guanosine 3' 5'-monophosphate (cGMP)/nitric oxide (NO) pathway. Recently, some reports revealed the crosstalk between mLUTS/BPH and endothelial dysfunction in microvessels [10,11]. MiRNAs are small, ncRNAs of 21-25 nucleotides that regulate gene expression via translational inhibition or activation of their target mRNAs. Various articles of research have indicated that some miRNAs play important roles in modulating the progress on endothelial dysfunction associated with atherosclerosis and ischemia [12]. In this study, we focused on three miRNAs (miR-21-5p [13,14], miR-126-5p [15] \& miR-155-5p [16]) that possibly regulate the function of endothelial cells via the control of several genes. We investigated whether the expression pattern of these urine miRNAs is associated with the clinical effects in the mLUTS/BPH patients treated with tadalafil.

\section{Patients \& methods}

\section{Study population \& urine collection}

This clinical study protocol was approved by the institutional review board of Osaka City University. A total of 70 patients with mLUTS caused by BPH were enrolled in this study between 2014 and 2016. The ethics committees of the participating hospitals approved this study, and informed consent was obtained from each patient. Finally, a total of 55 patients, excluding the patients who discontinued the administration, were examined. The patients received the administration of a PDE5 inhibitor, tadalafil, $5 \mathrm{mg} / \mathrm{day}$ for 12 weeks and their spot urine was used for measurement of the expression of miRNAs before and at 4 weeks after the treatment. Each spot urine was collected in the urine collection and preservation tube (Norgen Bioteck Co., Ontario, Canada) and stored at room temperature before isolation of miRNAs.

\section{Evaluation of mLUTS in subjective \& objective findings \& definition of clinical response}

Subjective urinary symptom score (International Prostate Symptom Score [I-PSS] and Overactive Bladder Symptoms Score [OABSS]), QoL and Nocturia-QoL index were estimated before and at 4, 8 and 12 weeks after administration of tadalafil. Objective urinary parameters (maximum urinary flow rate $\left[Q_{\max }\right]$ and average urinary flow rate $\left[\mathrm{Q}_{\mathrm{ave}}\right]$ ) in the uroflowmetry were measured before and at 12 weeks after the treatment. The clinically meaningful improvement (CMI) was defined as $\geq 25 \%$ baseline-to-endpoint total IPSS improvement; or $\geq 2.5$ $\mathrm{ml} / \mathrm{s}$ baseline-to-endpoint $\mathrm{Q}_{\max }$ improvement.

\section{RNA isolation \& reverse transcription \& real-time polymerase chain reaction}

Caenorhabditis elegans miR-39 (cel-miR-39) was used as external reference for assaying the miRNAs in urine [17]. Cel-miR-39 of $20 \mathrm{pmol} / 1$ was added as the spin-in control after the denaturing solution of $500 \mu$ was added. Total RNA including miRNAs component was isolated and purified using the urine MicroRNA Purification Kit (Norgen Bioteck Co.) in accordance with the manufacturer's protocol. The reverse transcription was performed with 10 ng of total RNA and TaqMan MicroRNA Reverse Transcription Kit (Applied Biosystems, CA, USA). The target miRNA was quantified using TaqMan Universal Master Mix II (no UNG) and each TaqMan assay; has-miR-21-5p (ID: 000397), has-miR-126-5p (ID: 000451) and has-miR-155-5p (ID: 002623), according to the manufacturer's protocol (Applied Biosystems). Each reaction was carried out in a total volume of $20 \mu$ l containing $1.3 \mu \mathrm{l}$ reverse transcription products, $1 \mu \mathrm{l} 20 \times$ TaqMan assay primer, $10 \mu \mathrm{l} 2 \times$ TaqMan Universal Master Mix II 
Table 1. Clinical characteristics and parameters of the patients.

\begin{tabular}{|c|c|c|c|}
\hline Variable & Responder $(n=36)$ & Nonresponder $(n=19)$ & p-value \\
\hline Age (years) & $68.5 \pm 1.6$ & $72.7 \pm 1.6$ & 0.069 \\
\hline BMI $\left(\mathrm{kg} / \mathrm{m}^{2}\right)$ & $24.1 \pm 0.6$ & $24.0 \pm 0.6$ & 0.4 \\
\hline PV (ml) & $30.0 \pm 4.3$ & $33.3 \pm 5.9$ & 0.43 \\
\hline \multicolumn{4}{|l|}{ I-PSS } \\
\hline Total score & $18.4 \pm 1.5$ & $16.3 \pm 1.6$ & 0.32 \\
\hline Storage subscore & $8.2 \pm 0.7$ & $7.1 \pm 0.7$ & 0.34 \\
\hline Voiding subscore & $10.2 \pm 1.0$ & $9.2 \pm 1.3$ & 0.48 \\
\hline QoL & $4.7 \pm 0.2$ & $4.5 \pm 0.2$ & 0.41 \\
\hline OABSS & $6.1 \pm 0.7$ & $5.2 \pm 0.6$ & 0.48 \\
\hline N-QoL index & $15.3 \pm 2.2$ & $15.4 \pm 1.9$ & 0.82 \\
\hline \multicolumn{4}{|l|}{ BPH severity } \\
\hline Mild & 5 & 3 & 0.76 \\
\hline Moderate & 16 & 10 & \\
\hline Severe & 15 & 6 & \\
\hline \multicolumn{4}{|c|}{ Additional use of tadalafil on $\alpha 1$ adrenoceptor blocker } \\
\hline No & 14 & 8 & 0.82 \\
\hline Yes & 22 & 11 & \\
\hline
\end{tabular}

(no UNG) and nuclease-free water to adjust the volume. The PCR reaction was performed as follows: $95^{\circ} \mathrm{C}$ for 10 min, followed by 50 cycles of $95^{\circ} \mathrm{C}$ for $15 \mathrm{~s}$ and $60^{\circ} \mathrm{C}$ for $30 \mathrm{~s}$. The comparative cycle threshold method $(\Delta \mathrm{Ct})$ was exploited to calculate the relative expression level of miRNA. Mean Ct values and deviations between the duplicates were calculated for all samples. $\Delta \mathrm{Ct}=\mathrm{Ct}$ (target miRNA) - $\mathrm{Ct}$ (Cel-miR-39) and relative miRNA expression were determined using the formula $2-\Delta \mathrm{Ct}$. In addition, each miRNA expression was set as the ratio against each urine creatinine level to avoid bias of urine concentration. The final values of miRNA levels were in logarithm scale.

\section{Statistical analysis}

Statistical analysis was performed by the Ekuseru-Toukei 2015 software (Social Survey Research Information Co., Ltd, Tokyo, Japan). The nonparametric Mann-Whitney $U$ test and $\chi^{2}$ test were used to analyze differences in the clinical characteristics and miRNAs abundances in two groups. The influence of explanatory variables on CMI was analyzed by means of the logistic regression analysis. Receiver operating characteristics curve was applied to analyze the prognostic value of urine miR-21-5p. Youden Index (sensitivity and 1-specificity) was used to identify the optimal cut-off threshold value. p-values $<0.05$ were considered statistically significant.

\section{Results}

\section{Basic characteristics of BPH patients}

Table 1 shows the clinical characteristics of responders $(n=36)$ and nonresponders $(n=19)$ based on the definition of CMI related with tadalafil treatment. There are no significant differences between two groups in age, BMI, prostate volume, total I-PSS, I-PSS voiding and storage subscore, QoL, Nocturia-QoL, OABSS, the severity grade of $\mathrm{BPH}$ and the rate of additional use on $\alpha 1$ adrenoceptor blocker.

\section{Comparison of responders \& nonresponders regarding overactive bladder symptoms}

As shown in Figure 1, the OABSS values of responders were significantly reduced by treatment with tadalafil, but those of nonresponders were not significantly changed. The responders satisfied with CMI criteria also showed significant improvement in the scale of overactive bladder. 

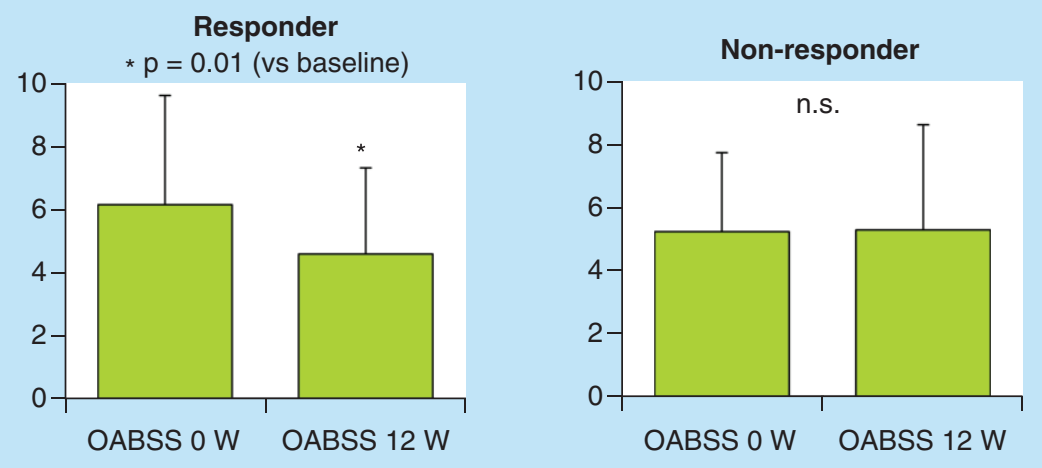

Figure 1. In the clinically meaningful improvement responder group, the values of Overactive Bladder Symptoms Score in post-treatment 12 weeks were significantly lower than those in pretreatment $\mathbf{0}$ week. In the clinically meaningful improvement nonresponder group, there was no significant difference between pre- and post-treatment 12 weeks Overactive Bladder Symptoms Score values.

n.s.: Not significant; OABSS: Overactive Bladder Symptoms Score.

\begin{tabular}{l}
$\begin{array}{l}\text { Table 2. Possible predictors in response to tadalafil treatment - logistic univariate and multivariate regression analyses. } \\
\text { Variables }\end{array}$ \\
\cline { 2 - 5 }
\end{tabular}

$\Delta$ : Change between baseline and post-treatment levels of miR-21-5p (increased vs decreased/unchanged); OR: Odds ratio.

Comparison of responders \& nonresponders in the baseline levels of urine miRNAs

The results showed that the baseline urine levels of miR-21-5p, miR-126-5p and miR-155-5p in responders were significantly lower compared with the baseline urine levels of them in nonresponders, respectively $(p<0.001$, $\mathrm{p}=0.01$ and $\mathrm{p}=0.006$ (Figure $2 \mathrm{~A})$.

Comparison of responders \& nonresponders in the changes between the baseline \& post-treatment levels of urine miRNAs

The change of urine miR-21-5p expression between the baseline and post treatment 4 weeks in responders was significantly high compared with that in nonresponders $(\mathrm{p}<0.01)$ (Figure $2 \mathrm{~B})$. After tadalafil treatment, responders tended to have an increase in post-treatment levels of urine miR-21-5p. There are no significant differences between responders and nonresponders in alterations of either urine miR-126-5p or miR-155-5p due to tadalafil administration, respectively $(\mathrm{p}=0.22,0.06)$ (Figure 2B).

Univariate \& multivariate repression analyses of parameters associated with urine miRNAs expression

First, there is a no significant impact factor in several clinical parameters (i.e., age, BMI, prostate volume, I-PSS, OABSS, QOL, etc.) in univariate analysis. As shown in Table 2, the results showed that each baseline levels in urine miR-21-5p, miR-126-5p and miR-155-5p and the ratio of post-/pretreatment urine levels of miR-21-5p have a significant impact on CMI of treatment with tadalafil in univariate models. Furthermore, the multivariate analysis confirmed the baseline levels in urine miR-21-5p as an independent predictor (OR: 0.28; 95\% CI: 0.10-0.77; $\mathrm{p}=0.014)$.

Predictive value of urine miRNAs in mLUTS/BPH patients treated with tadalafil

We performed receiver operating characteristics analysis to evaluate the predictive power of the baseline urine miRNAs levels for treatment response of tadalafil against mLUTS/BPH patients. The baseline urine miR-21-5p 

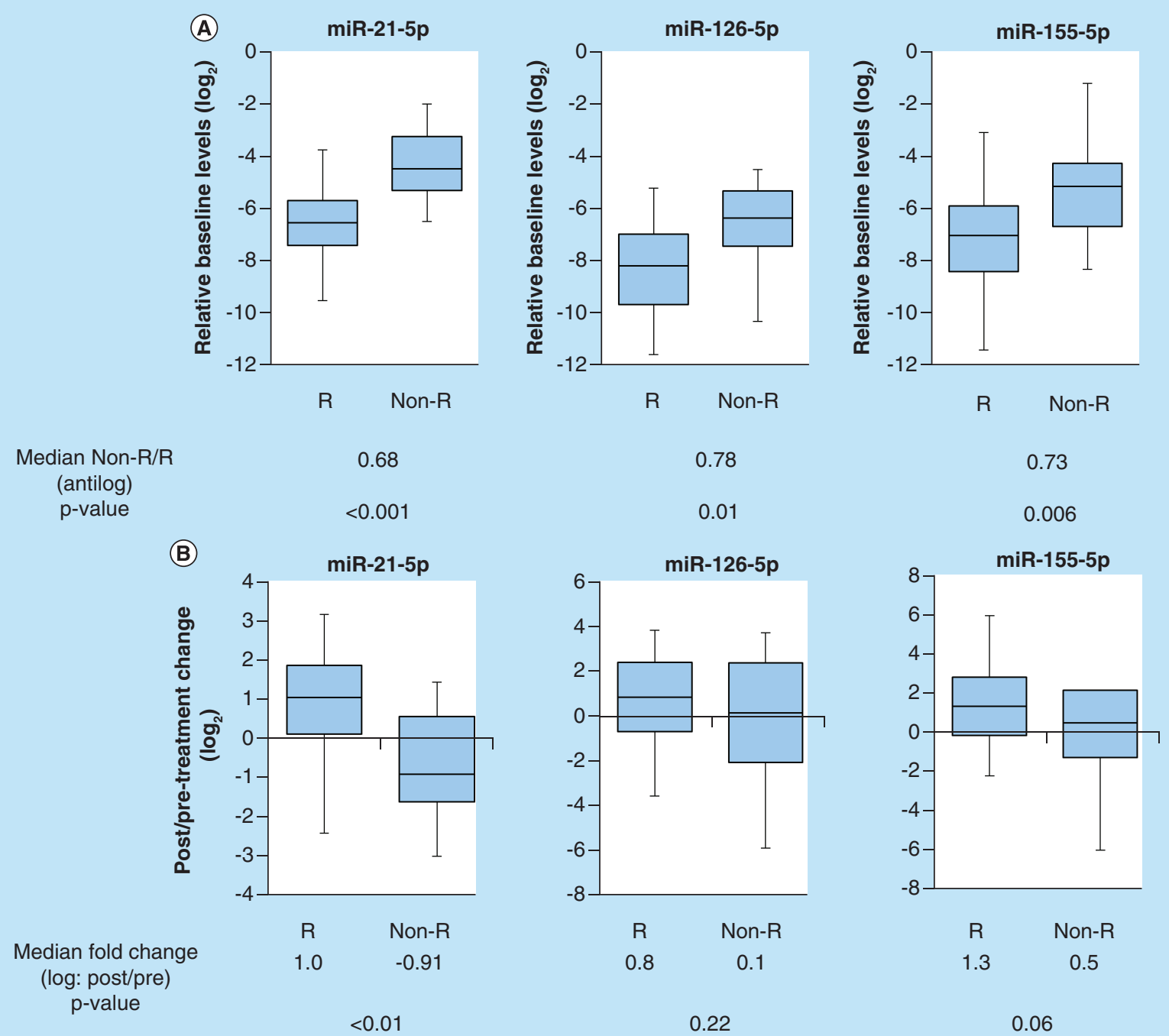

Figure 2. Comparison of responders and nonresponders in urine levels of miR-21-5p, miR-126-5p and miR-155-5p. (A) The baseline urine levels of miR-21-5p, miR-126-5p and miR-155-5p in responders were significantly lower compared with the baseline urine levels of them in nonresponders, respectively $(p<0.001, p=0.01$ and $p=0.006$ ). (B) The change between the pre- and post-treatment 4 weeks in urine miR-21-5p levels of responders was significantly high compared with that of nonresponders $(p<0.01)$. The change between the pre- and post-treatment 4 weeks in either urine miR-126-5p or miR-155-5p levels of responders showed no significant difference from that of nonresponders, respectively.

Non-R: Non-responder; R: Responder.

yielded AUC of 0.85 (95\% CI: 0.75-0.95; p < 0.001) and the optimal cut-off value was -5.7 with sensitivity and specificity of 78.8 and $78.9 \%$, respectively (Figure 3 ). In addition, the baseline urine miR-126-5p and miR-155-5p exhibited AUC of $0.73(95 \%$ CI: $0.58-0.88$; sensitivity $=72.7 \%$, specificity $=68.4 \%, p=0.003)$ and $0.73(95 \%$ CI: $0.59-0.87$; sensitivity $=84.8 \%$, specificity $=57.9 \%, p=0.002)$, respectively (Supplementary Figure 1$)$.

\section{Discussion}

The PDE5-Is have been reported to exert strong pharmaceutical effects for smooth muscle relaxant in bladder neck and prostatic urethra $[9,18,19]$, vasodilatation response in lower urinary tract (LUT) $[7,20]$, inhibition of bladder afferent nerve activity [21] and reduction in prostatic inflammation related with LUTS [8]. Although the functional role of PDE5 remains controversial, a main stream is NO/cGMP signaling pathway in the crosstalk between efferent nerve terminal and LUT smooth muscle cell, or endothelial and vessel smooth muscle cell [22]. Previous reports revealed that expression of PDE5 is robust in endothelial and smooth muscle cells of LUT vessels, and 


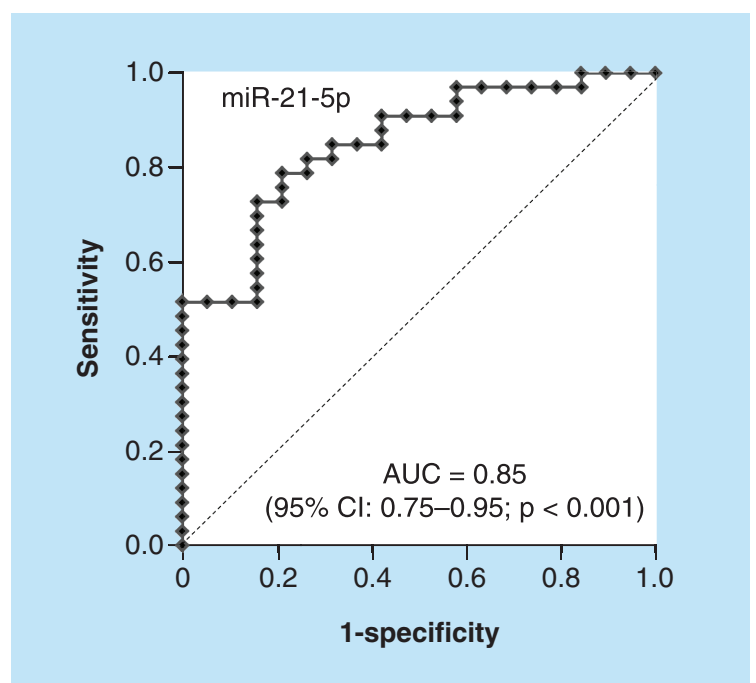

Figure 3. The baseline urine miR-21-5p produced AUC of 0.85 (95\% Cl: $0.75-0.95 ; p<0.001)$ and the optimal cut-off value was -5.7 with sensitivity and specificity of 78.8 and $\mathbf{7 8 . 9} \%$, respectively. AUC: Area under curve.

tadalafil plays a critical role in vasodilatation of vesicular-differential artery via inhibition of cGMP degradation by blocking of PDE5 activity [6,23]. PDE5-Is initially have been used on-demand in erectile dysfunction (ED) for the purpose of vasodilatation of penile cavernous arteries. Administration of tadalafil for ED patients was also reported to increase percentage flow-mediated dilatation as an indicator of endothelial function and induce circulating endothelial precursor cells $[24,25]$. In addition, a recent study reported that response of endothelium to tadalafil treatment significantly correlated to improvement of mLUTS [26].

While there is a large amount of scientific evidence for clinical response of PDE5-Is in mLUTS and ED, few reports investigating which subgroup of patients with mLUTS would represent preferable effectiveness of PDE5-Is have been found until now. If an appropriate biomarker for predicting the clinical response of PDE5-Is could be put into practical use, it would contribute to a development of personalized medical care for mLUTS/BPH.

To our knowledge, this study is the first report to explore whether urine levels of miRNAs associated with regulation of endothelial functions could predict the response to the PDE5-I tadalafil in mLUTS/BPH. Our study demonstrated that the pretreatment baseline levels of three urine miRNAs, and the change between the pre- and post-treatment level in urine miR-21-5p could differentiate responders from nonresponders to tadalafil. Finally, we provided evidence that the baseline urine levels of miR-21-5p exerted an independent predictive factor in logistic multivariate repression analysis. It has been reported that the expression of miR-21 is induced by blood shear stress in endothelium, and miR-21 increases the production of NO through upregulation of endothelial NO synthase and suppresses apoptosis of endothelium via inhibition of phosphate and tensin homolog deleted from chromosome 10 [13,14]. In a past study, it was demonstrated that reduction of endothelial-expressed miRNAs may be attributed to uptake into atherosclerotic lesions in patients with cardiovascular disease [27,28]. The population of patients that have endothelial dysfunction in LUT microvessels concomitantly with low levels of circulating endothelial miRNAs may be a good candidate for treating with tadalafil. On the contrary, Vlachopoulos et al. reported that mLUTS/BPH patients with cardiovascular disease risk, who were administrated $>1$ antihypertensive drug, have significantly lower IPSS improvement compared with men taking $\leq 1 \mathrm{drug}$ [29]. Furthermore, they demonstrated that use of diuretics resulted in significantly lower IPSS improvement compared with men taking other antihypertensives or no drugs. The patients with severe atherosclerosis, who need some kinds of antihypertensive drugs, seem to be inappropriate for treatment with tadalafil because of irreversible endothelial dysfunction. Moreover, upregulation of miR-21 has been shown to lead to MAPK-dependent reactive oxygen species production and reduction of NO bioavailability in endothelial precursor cells [30]. Interestingly, antagonism of miR-21 was reported to improve dysfunction of angiogenic progenitor cells [31].

Although this study indicated that the baseline levels of urine miR21-5p below the cutoff -5.7 with sensitivity and specificity of 78.8 and $78.9 \%$, respectively, may be a useful predictor for tadalafil treatment in mLUTS/BPH, there are some limitations of this study including no examination was performed with negative control miRNAs that do not target endothelium; numbers of examined BPH patients; and no measurements for clinical endothelial function values (e.g., percentage flow-mediated dilatation, reactive hyperemia index) or the blood flow velocity 
surrounding bladder in researched patients. Based on the current data, we will prospectively investigate whether urine miR-21-5p levels correlate with the values in endothelial function and vesical blood flow in BPH patients treated with tadalafil.

\section{Conclusion}

In summary, we confirmed that urine expression patterns of miRNAs associated with endothelial function possibly correlate with effectiveness of tadalafil treatment in mLUTS/BPH patients. Particularly, the baseline urine levels of miR-21-5p could be a promising biomarker predictive for its response.

\section{Future perspective}

All evidence from this study showed that urine levels of three endothelium-associated miRNAs (miR-21-5p, miR126-5p, miR-155-5p) have a potential for correlation with the treatment response to tadalafil in mLUTS/BPH patients. In particular, the baseline urine level of miR-21-5p exerted an excellent prediction for effectiveness of tadalafil in those patients. The next step is to determine whether urine levels of these miRNAs also associate with endothelial function values and vesical blood circulation in a large number of patients. Finally, the determination of appropriate cut-off values of urine levels in endothelial miRNAs will lead to a tailor-made medication of tadalafil for mLUTS/BPH patients.

\section{Summary points}

- Each baseline urine level of miR-21-5p, miR-126-5p and miR-155-5p in tadalafil responders is significantly lower than those in tadalafil nonresponders, respectively.

- Change between baseline and post-treatment urine level of miR-21-5p in tadalafil responders is significantly higher than that in tadalafil nonresponders.

- Baseline urine levels of miR-21-5p are an independent factor predictive for tadalafil response.

- Baseline urine miR-21-5P yields AUC of 0.85 (95\% Cl: $0.75-0.95 ; p<0.001)$ and the optimal cut-off value is -5.7 with sensitivity and specificity of 78.8 and $78.9 \%$, respectively.

\section{Supplementary Data}

To view the supplementary data that accompany this paperplease visit the journal website at: www.futurescience.com/doi/full/10.4155/fsoa-2018-0012

\section{Author's contributions}

T Tanaka had a role in study concept and design, analysis and interpretation of data and preparation of manuscript; A Minami had a role in accomplishment of experiments and data analysis; K Tashiro, N Yoshida, A Tohda, Y Yamakoshi, R Yasumoto and S Sugita had a role in collecting patients' information and urine samples; T Nakatani has a role in study concept and consultation on the experimental matter.

\section{Acknowledgements}

The authors thank S Masaki for her assistance in measurement for miRNAs expressions in collected urine samples.

\section{Financial \& competing interests disclosure}

The authors have no relevant affiliations or financial involvement with any organization or entity with a financial interest in or financial conflict with the subject matter or materials discussed in the manuscript. This includes employment, consultancies, honoraria, stock ownership or options, expert testimony, grants or patents received or pending, or royalties.

No writing assistance was utilized in the production of this manuscript.

\section{Ethical conduct of research}

The authors state that they have obtained appropriate institutional review board approval or have followed the principles outlined in the Declaration of Helsinki for all human or animal experimental investigations. In addition, for investigations involving human subjects, informed consent has been obtained from the participants involved. 
Open access

This work is licensed under the Creative Commons Attribution 4.0 License. To view a copy of this license, visit http://creativecomm ons.org/licenses/by/4.0/

\section{References}

Papers of special note have been highlighted as: $\bullet$ of interest; $\bullet \bullet$ of considerable interest

1 Hammarsten J, Hogstedt B, Holthuis N, Mellstrom D. Components of the metabolic syndrome-risk factors for the development of benign prostatic hyperplasia. Prostate Cancer Prostatic Dis. 1(3), 157-162 (1998).

2 De Nunzio C, Aronson W, Freedland SJ, Giovannucci E, Parsons JK. The correlation between metabolic syndrome and prostatic diseases. Eur. Urol. 61(3), 560-570 (2012).

3 De Nunzio C, Cindolo L, Gacci M et al. Metabolic syndrome and lower urinary tract symptoms in patients with benign prostatic enlargement: a possible link to storage symptoms. Urology 84(5), 1181-1187 (2014).

- Metabolic syndrome is closely associated with pathophysiology of benign prostatic hyperplasia (BPH)

4 Gratzke C, Bachmann A, Descazeaud A et al. EAU guidelines on the assessment of non-neurogenic male lower urinary tract symptoms including benign prostatic obstruction. Eur. Urol. 67(6), 1099-1109 (2015).

5 Homma Y, Gotoh M, Kawauchi A et al. Clinical guidelines for male lower urinary tract symptoms and benign prostatic hyperplasia. Int. J. Urol. 24(10), 716-729 (2017).

- Tadalafil is worldwide defined as an excellentagent for treating male lower urinary tract symptoms (mLUTS)/BPH.

6 Morelli A, Sarchielli E, Comeglio P et al. Phosphodiesterase type 5 expression in human and rat lower urinary tract tissues and the effect of tadalafil on prostate gland oxygenation in spontaneously hypertensive rats. J. Sex. Med. 8(10), 2746-2760 (2011).

7 Yoshinaga R, Kawai Y, Oka M, Fuchikami C, Oyama T. Effect of a single treatment with tadalafil on blood flow in lower urinary tract tissues in rat models of bladder overdistension/emptying and abdominal aorta clamping/release. Eur. J. Pharmacol. 754, 92-97 (2015).

8 Vignozzi L, Gacci M, Cellai I et al. PDE5 inhibitors blunt inflammation in human BPH: a potential mechanism of action for PDE5 inhibitors in LUTS. Prostate 73(13), 1391-1402 (2013).

9 Angulo J, Cuevas P, Fernandez A et al. Tadalafil enhances the inhibitory effects of tamsulosin on neurogenic contractions of human prostate and bladder neck. J. Sex. Med. 9(9), 2293-2306 (2012).

10 Cellek S, Cameron NE, Cotter MA, Fry CH, Ilo D. Microvascular dysfunction and efficacy of PDE5 inhibitors in BPH-LUTS. Nat. Rev. Urol. 11(4), 231-241 (2014).

11 Amano T, Earle C, Imao T, Matsumoto Y, Kishikage T. Administration of daily $5 \mathrm{mg}$ tadalafil improves endothelial function in patients with benign prostatic hyperplasia. Aging Male doi:10.1080/13685538.2017.1367922 (2017) (Epub ahead of print).

- PDE5-Is has multiple pharmaceutical effects onrelief of LUTS.

12 Raitoharju E, Oksala N, Lehtimaki T. MicroRNAs in the atherosclerotic plaque. Clin. Chem. 59(12), 1708-1721 (2013).

13 Rippe C, Blimline M, Magerko KA et al. MicroRNA changes in human arterial endothelial cells with senescence: relation to apoptosis, eNOS and inflammation. Exp. Gerontol. 47(1), 45-51 (2012).

14 Weber M, Baker MB, Moore JP, Searles CD. MiR-21 is induced in endothelial cells by shear stress and modulates apoptosis and eNOS activity. Biochem. Biophys. Res. Commun. 393(4), 643-648 (2010).

15 Schober A, Nazari-Jahantigh M, Wei Y et al. MicroRNA-126-5p promotes endothelial proliferation and limits atherosclerosis by suppressing Dlk1. Nat. Med. 20(4), 368-376 (2014).

16 Zhu N, Zhang D, Chen S et al. Endothelial enriched microRNAs regulate angiotensin II-induced endothelial inflammation and migration. Atherosclerosis 215(2), 286-293 (2011).

17 Sourvinou IS, Markou A, Lianidou ES. Quantification of circulating miRNAs in plasma: effect of preanalytical and analytical parameters on their isolation and stability. J. Mol. Diagn. 15(6), 827-834 (2013)

-• MiRNAs crucially regulate endothelial functionsin atherosclerosis.

18 Uckert S, Sormes M, Kedia G et al. Effects of phosphodiesterase inhibitors on tension induced by norepinephrine and accumulation of cyclic nucleotides in isolated human prostatic tissue. Urology 71(3), 526-530 (2008).

19 Oger S, Behr-Roussel D, Gorny D et al. Signalling pathways involved in sildenafil-induced relaxation of human bladder dome smooth muscle. Br. J. Pharmacol. 160(5), 1135-1143 (2010).

20 Kawai Y, Oka M, Yoshinaga R, Fuchikami C, Oyama T. Effects of the phosphodiesterase 5 inhibitor tadalafil on bladder function in a rat model of partial bladder outlet obstruction. Neurourol. Urodyn. 35(4), 444-449 (2016).

21 Caremel R, Oger-Roussel S, Behr-Roussel D, Grise P, Giuliano FA. Nitric oxide/cyclic guanosine monophosphate signalling mediates an inhibitory action on sensory pathways of the micturition reflex in the rat. Eur. Urol. 58(4), 616-625 (2010).

22 Higashi Y. Lower urinary tract symptoms/benign prostatic hypertrophy and vascular function: role of the nitric oxide-phosphodiesterase type 5-cyclic guanosine $3^{\prime}, 5^{\prime}$-monophosphate pathway. Int. J. Urol. 24(6), 412-424 (2017). 
23 Fibbi B, Morelli A, Vignozzi L et al. Characterization of phosphodiesterase type 5 expression and functional activity in the human male lower urinary tract. J. Sex. Med. 7(1 Pt 1), 59-69 (2010).

- PDE5-Is have ameliorative effects in someregions of LUT.

24 Foresta C, Ferlin A, De Toni L et al. Circulating endothelial progenitor cells and endothelial function after chronic tadalafil treatment in subjects with erectile dysfunction. Int. J. Impot. Res. 18(5), 484-488 (2006).

25 Rosano GM, Aversa A, Vitale C, Fabbri A, Fini M, Spera G. Chronic treatment with tadalafil improves endothelial function in men with increased cardiovascular risk. Eur. Urol. 47(2), 214-220; discussion 220-212 (2005).

26 Fukumoto K, Nagai A, Hara R, Fujii T, Miyaji Y. Tadalafil for male lower urinary tract symptoms improves endothelial function. Int. J. Urol. 24(3), 206-210 (2017).

-• Tadalafil markedly improves erectile dysfunction and LUTS withendothelial dysfunction.

27 Jamaluddin MS, Weakley SM, Zhang L et al. miRNAs: roles and clinical applications in vascular disease. Expert Rev. Mol. Diagn. 11(1), 79-89 (2011).

28 Fichtlscherer S, De Rosa S, Fox H et al. Circulating microRNAs in patients with coronary artery disease. Circ. Res. 107(5), 677-684 (2010).

-. Circulating miRNAs represents clinical conditionin CVD.

29 Vlachopoulos C, Oelke M, Maggi M et al. Impact of cardiovascular risk factors and related comorbid conditions and medical therapy reported at baseline on the treatment response to tadalafil $5 \mathrm{mg}$ once-daily in men with lower urinary tract symptoms associated with benign prostatic hyperplasia: an integrated analysis of four randomised, double-blind, placebo-controlled, clinical trials. Int. J. Clin. Pract. 69(12), 1496-1507 (2015).

-. Treatment of tadalafil seems preferable for LUTS with atherosclerosis to some extent.

30 Fleissner F, Jazbutyte V, Fiedler J et al. Short communication: asymmetric dimethylarginine impairs angiogenic progenitor cell function in patients with coronary artery disease through a microRNA-21-dependent mechanism. Circ. Res. 107(1), 138-143 (2010).

31 Quintavalle M, Condorelli G, Elia L. Arterial remodeling and atherosclerosis: miRNAs involvement. Vascul. Pharmacol. 55(4), 106-110 (2011).

-. MiR-21 is a potential target to treatatherosclerosis and CVD. 
